\title{
Otimização do dimensionamento em sistemas de bombeamento fotovoltaico: validação de modelo em sistema piloto na comunidade rural de Rio Belo, Orleans (SC)
}

Sizing optimization in Solar Photovoltaic Water Pumping System: model validation in a pilot unit located in the rural community of Rio Belo, Orleans/SC, Brazil

\author{
Rodrigo Delalibera Carvalho"* $₫$, Ramon Lucas Dalsasso' ${ }^{\circledR}$, \\ Tiago Lemos Guedes' ${ }^{1}$, Joana Andreazza Claudino dos Santos ${ }^{1}$
}

\section{RESUMO}

A gestão dos recursos hídricos e energéticos é o principal desafio que a sociedade contemporânea enfrenta atualmente. Água e energia são fatores fundamentais para o desenvolvimento socioeconômico das comunidades, principalmente aquelas situadas em locais remotos, e, nesse cenário, a falta de energia é o aspecto mais preocupante. Os sistemas de bombeamento fotovoltaico apresentam vantagens para a inserção em comunidades isoladas, como fácil instalação e pouca manutenção durante o ciclo de vida, porém o dimensionamento inadequado pode gerar gastos desnecessários ou comprometer o abastecimento de água. Simular o desempenho favorece a concepção de sistemas e permite alcançar a otimização técnica e econômica em projetos da referida tecnologia. O estudo propõe um modelo matemático de simulação e a utilização do generalized reduced gradient (GRG) e de dados regionais de radiação solar, de consumo de água, de produtividade de poços e de capacidade do sistema de bombeamento para obter a referida otimização. A validação do respectivo modelo foi realizada em um sistema piloto, localizado em uma comunidade rural no município de Orleans, no Estado de Santa Catarina. A localidade apresenta uma das menores médias de radiação anual no país, aproximadamente $4,5 \mathrm{kWh} \cdot \mathrm{m}^{2} / \mathrm{dia}$. A simulação efetuada nas condições naturais do sistema piloto mostrou resultados bem próximos do comportamento monitorado, evidenciando, assim, a eficiência do modelo. O uso da ferramenta auxilia no processo de tomada de decisão quanto à concepção de projetos, além de beneficiar as comunidades com acesso à água e à energia, trazendo benefícios em todas as esferas: social, ambiental e econômica.

Palavras-chave: bombeamento fotovoltaico; modelo; dimensionamento.

\section{ABSTRACT}

The management of water and energy resources are the main challenges that contemporary society faces nowadays. Water and energy are fundamental factors for socio-economic development of the communities, especially those in remote locations, and in this case the lack of energy is the most worrying aspect. Solar photovoltaic pumping systems have advantages for insertion in isolated communities, such as easy installation and low maintenance during the life cycle, but inappropriate sizing can create unnecessary costs or affect the water supply. Simulating performance favors the system's sizing and allows achieving technical and economic optimization of these technology projects. This study proposes a mathematical mode of simulation, use of the Generalized Reduced Gradient (GRG), solar radiation regional data, and water consumption, wells' productivity and pumping system capacity to obtain this optimization. The mode was validated in a pilot system, located in a rural community in the city of Orleans, State of Santa Catarina, Brazil. The place has one of the lowest average annual radiation in the country, approximately $4.5 \mathrm{kWh} / \mathrm{m}^{2}$.day. The simulation performed in the natural pilot system conditions presented results very close to the monitored behavior, thus demonstrating its efficiency. Using this tool assists in the decisionmaking process in designing projects and benefits the communities with access to water and energy, bringing benefits in all spheres: social, environmental and economic

Keywords: solar water pump; simulation; sizing. 


\section{INTRODUÇÃO}

Os acessos à água potável e à energia limpa e rentável são 2 dos 17 Objetivos do Desenvolvimento Sustentável, propostos pela Organização das Nações Unidas (UNITED NATIONS, 2015).

As águas subterrâneas, após a cloração, geralmente atendem ou têm qualidade próxima dos padrões de potabilidade. Ao longo do território brasileiro, podem ser encontradas diversas fontes com qualidade adequada, entretanto a falta de energia elétrica dificulta o acesso a esse recurso, especialmente em comunidades isoladas.

O transporte de água potável por meio de fontes renováveis (i.e., sol, vento) tem se mostrado uma técnica promissora em regiões remotas e com disponibilidade desses recursos naturais, a exemplo de aplicações em áreas desérticas no Marrocos, na ilha de Caiapós, em São Romão (MG) (BOITRAGO et al., 2016), em áreas rurais na Zâmbia (SETIAWAN et al., 2014), entre outros.

A busca pelo melhor aproveitamento dos recursos naturais e o consequente aperfeiçoamento no dimensionamento de sistemas de bombeamento fotovoltaico têm sido objetos de estudos relacionados ao tema, descritos adiante.

O desempenho de um sistema de bombeamento fotovoltaico pode ser avaliado por meio de um programa de simulação, no qual são considerados diversos parâmetros inerentes ao sistema, como respectiva localização geográfica, radiação solar, padrão de consumo de água e aspectos hidrogeológicos. Assim, são revisadas as metodologias e ferramentas computacionais para previsão e avaliação de desempenho, aplicadas em vários estudos.

Gad (2009) desenvolveu uma metodologia através de um programa de computador para simular o desempenho de um sistema fotovoltaico de bombeamento de água para uso doméstico no Egito. O programa simulou, hora após hora, o desempenho do sistema durante cada dia do ano, com orientações potencializadas dos módulos fotovoltaicos nas diferentes estações do ano. A eficiência calculada do conjunto fotovoltaico foi de $13,86 \%$ nos invernos e $13,91 \%$ nos verões. Kolhe, Joshi e Kothari (2004), por sua vez, ajustando manualmente os painéis solares três vezes ao dia, obtiveram eficiência $20 \%$ maior quando comparada a posição fixa.

Loxsom e Durongkaveroj (1994) criaram um algoritmo para avaliar o desempenho mensal de um sistema de bombeamento fotovoltaico. A partir da média mensal dos dados de insolação, é possível estimar o total do volume mensal de água bombeada utilizando um modelo de simulação horária.

Hadj Arab et al. (1991) analisaram o desempenho de configurações distintas de sistemas de bombeamento fotovoltaico em quatro diferentes localidades da Argélia utilizando dados do ano meteorológico padrão (typical metereological year - TMY). O estudo foi realizado com duas tecnologias de painéis fotovoltaicos, três capacidades de reservatório e diversas alturas manométricas aplicadas a duas bombas centrífugas. Para todas as configurações, concluíram que a localização geográfica influencia diretamente no desempenho do sistema. Nesse estudo, regiões litorâneas apresentaram menor desempenho que regiões desérticas, atribuído a maior presença de nuvens, logo, períodos de baixa irradiação solar. Por outro lado, temperaturas elevadas no deserto podem comprometer o desempenho dos sistemas fotovoltaicos. Os custos dos geradores solares fotovoltaicos podem diminuir se o programa de simulação considerar os tipos de bomba utilizados, a altura manométrica envolvida e o perfil de carga diária. Portanto, o sistema pode ser aperfeiçoado com o estudo das necessidades individuais utilizando um programa de computador abalizado com modelos matemáticos.

Diferentes abordagens têm sido tratadas pelos pesquisadores, com o objetivo de buscar a solução ideal para o dimensionamento de um sistema de bombeamento fotovoltaico. $\mathrm{O}$ dimensionamento de sistemas fotovoltaicos autônomos consiste em encontrar as cargas elétricas necessárias com a menor média diária de insolação na superfície dos módulos fotovoltaicos, usualmente nos meses de inverno (PINHO \& GALDINO, 2014).

Para se alcançar a otimização de um sistema de abastecimento de água com o uso de energia fotovoltaica conforme tratado em Djurin, Margeta \& Glasnovic (2011), torna-se necessário analisar alguns fatores e a inter-relação existente entre eles, tais como as condições externas locais (temperatura e incidência de radiação solar), as características inerentes ao sistema de energia solar empregado (painel fotovoltaico, controlador de carga e inversor), as características da bomba e do poço em função da vazão $(\mathrm{Q})$ e as variações do volume do reservatório e do consumo diário de água. De acordo com Anis \& Norb (1994), três parâmetros são fundamentais para analisar o desempenho de um sistema: o tamanho dos módulos fotovoltaicos, a capacidade do banco de baterias e o volume do reservatório. A solução ideal é aquela que reduz o tamanho dos módulos fotovoltaicos, já que é o item de maior custo. Aumentar a capacidade do banco de baterias tem pouco efeito no desempenho do sistema.

Modelos matemáticos que utilizam o algoritmo não linear generalized reduced gradient (GRG) são rotineiramente empregados nas mais diversas áreas para a otimização de processos e avaliação de desempenho.

O presente estudo apresenta um modelo de simulação, de meia em meia hora, para avaliar o desempenho e aperfeiçoar o dimensionamento de um sistema acoplado de bombeamento fotovoltaico, isto é, sem banco de baterias. A validação do modelo foi realizada em um sistema piloto, localizado em uma comunidade rural, no município de Orleans, ao sul do Estado de Santa Catarina. A localidade está numa região que apresenta uma das menores médias de radiação anual no país, aproximadamente 4,0 kWh.m ${ }^{-2} / \mathrm{dia}$ (TIBA, 2000), todavia a disponibilidade e qualidade de águas subterrâneas encorajaram a aplicação da tecnologia de bombeamento fotovoltaico. 


\section{METODOLOGIA}

Para a realização deste trabalho, foram utilizados dados históricos, da literatura e relativos ao sistema piloto de bombeamento fotovoltaico, ilustrado pela Figura 1. O referido sistema foi construído numa propriedade rural, localizada na comunidade do Rio Belo, no município de Orleans (SC), com as seguintes características: um poço de produção, tipo escavado, diâmetro interno de $1 \mathrm{~m}$, com capacidade máxima de $2 \mathrm{~m}^{3} \cdot \mathrm{h}^{-1}$; três sistemas de bombeamento fotovoltaico, composto de painel fotovoltaico de $175 \mathrm{Wp}$, bomba submersa vibratória de $100 \mathrm{~W}$ e driver, com o objetivo de permitir variar o volume diário bombeado; um reservatório de distribuição com capacidade de 5.000 l; e um piranômetro.

Com o intuito de, opcionalmente, simular no sistema piloto o consumo de mais de uma residência, foi desenvolvido um dispositivo simulador de consumo. O aparato é composto de quatro válvulas solenoides acionadas por temporizadores analógicos programáveis (timers). Cada uma de suas quatro saídas possui orifício com diferente abertura, de forma que a combinação de válvulas abertas permite, a cada 30 minutos, obter o volume consumido equivalente ao de determinado número de residências, seguindo certo padrão de consumo. A parametrização do consumo da propriedade foi feita através de um estudo local, baseado na aplicação de um questionário sobre os hábitos de consumo de água de seus moradores, e instalação de um medidor eletromagnético com data logger na saída do reservatório da residência.
O período de coleta dos dados foi entre os meses de julho de 2014 e dezembro de 2015. O sistema piloto foi equipado com os seguintes sistemas de medição e registro de dados em data loggers: medidores eletromagnéticos de vazão, nas saídas das bombas e do reservatório, com registro de dados a cada 2 minutos; medidores de nível no poço e reservatório, com registro de dados a cada 15 minutos; e piranômetro, com registro de dados a cada 10 segundos. O modelo fornece a capacidade de um reservatório que atenda a determinada demanda de água, baseado no padrão de consumo, nas condições de radiação solar, na vazão de bombeamento e na capacidade de produção do poço. Os dados necessários para a aplicação do modelo são mencionados adiante. Para subsidiar o modelo, utilizou-se o mapa de radiação no plano inclinado, média sazonal (junho a agosto) do Atlas Brasileiro de Energia Solar (PEREIRA et al., 2006), ilustrado na Figura 2. Os menores valores de radiação no plano inclinado ocorrem nos meses de inverno em todo o território brasileiro.

Adicionalmente, foram utilizados dados de estações solarimétricas do Sistema de Organização Nacional de Dados Ambientais (SONDA), projeto do Instituto Nacional de Pesquisas Espaciais (INPE), referentes à média de radiação solar, durante o mês de julho, de estações solarimétricas de um município de cada região do território brasileiro, descritos a seguir: Palmas (Região Norte), São Luís (Região Nordeste), Brasília (Região Centro-Oeste), Cachoeira Paulista (Região Sudeste) e Florianópolis (Região Sul).

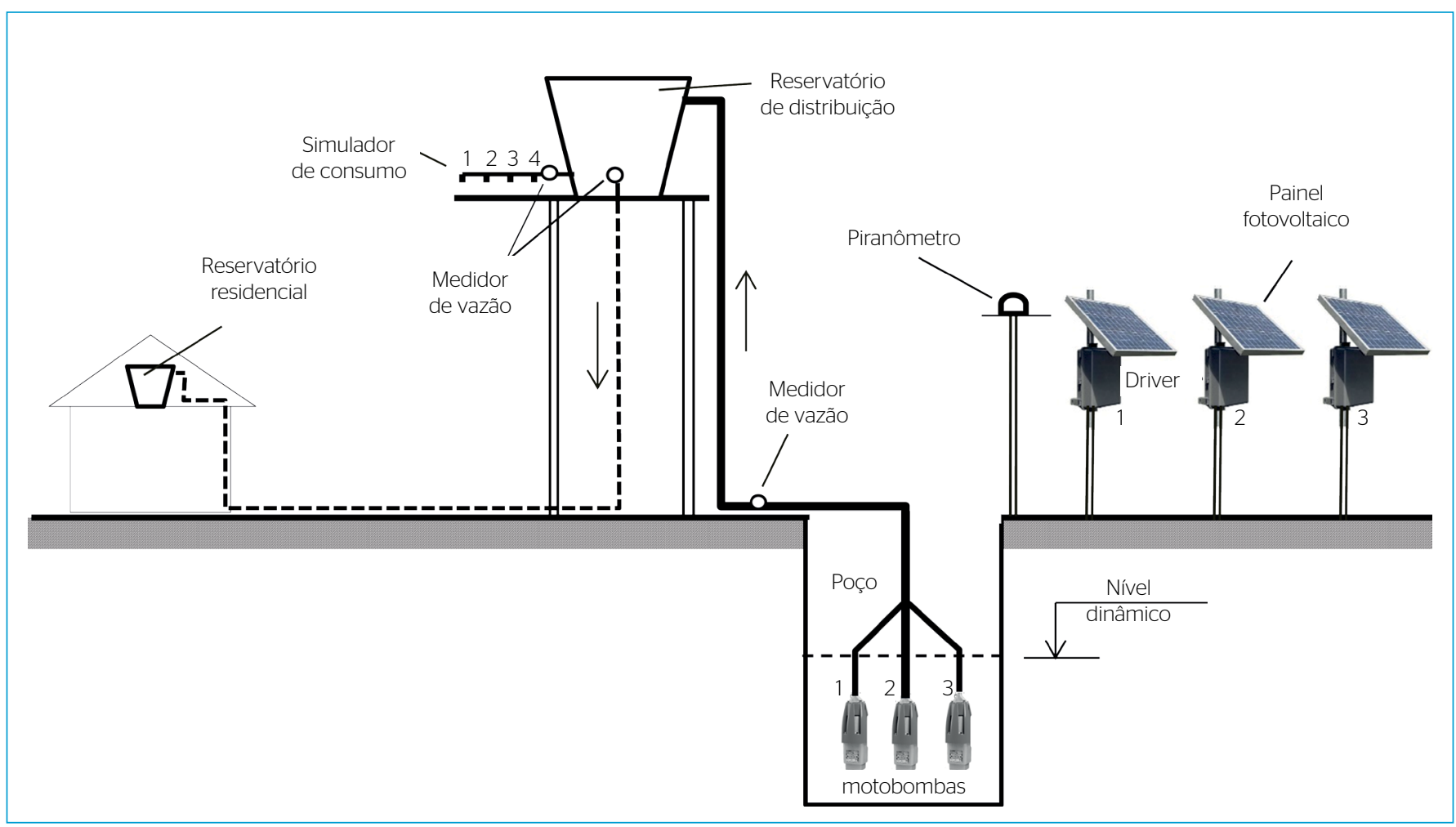

Figura 1 - Arranjo geral do sistema piloto. 
Através das curvas de radiação, foram estabelecidos os fatores horários de intensidade solar para cada região brasileira, a fim de estimar o bombeamento em diferentes intervalos diários em função da localidade. A Figura 3 ilustra o perfil de radiação solar de municípios brasileiros em cada região.

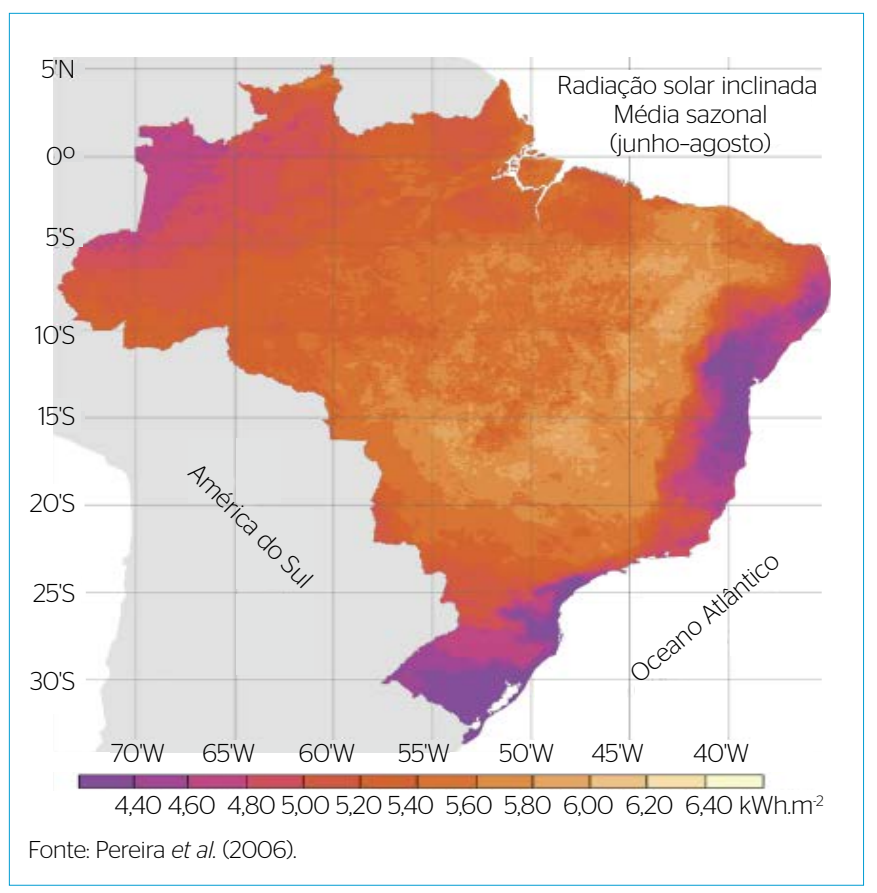

Figura 2 - Radiação solar inclinada nos meses de inverno.
O modelo também utiliza uma base de dados com diferentes configurações de conjuntos hidroenergéticos (bomba+painel). Após o processo de otimização, o referido modelo seleciona um subsistema de acordo com a vazão e altura manométrica.

No sistema piloto, foram utilizados três sistemas, independentes, compostos de bomba submersa vibratória solar de 100 $\mathrm{W}$, com módulos fotovoltaicos, e células tipo monocristalino (Mono-Si), de $175 \mathrm{Wp}$ cada. A energia elétrica proveniente do painel solar é fornecida à bomba através do dispositivo driver, que controla e atenua as variações de vazão em função das oscilações da radiação solar.

Em função da vazão necessária, pode ser considerada na simulação a utilização de número variado de poços, raso ou tubular profundo, cuja produção máxima deve ser informada, de acordo com as condições hidrogeológicas de cada região. Quanto aos reservatórios, são considerados dois materiais distintos em função do volume nominal: polietileno, para volumes de até $20 \mathrm{~m}^{3}$, e acima dessa quantidade, utiliza-se concreto - contudo é possível reconfigurar essa opção em função dos custos.

Conforme mencionado anteriormente, os modelos de simulação de desempenho são objetos de investigação no campo da tecnologia de bombeamento fotovoltaico. As pesquisas comumente utilizam intervalos horários. Com o intuito de avaliar a resiliência do sistema, o modelo proposto considera as condições mais críticas do ano, sendo

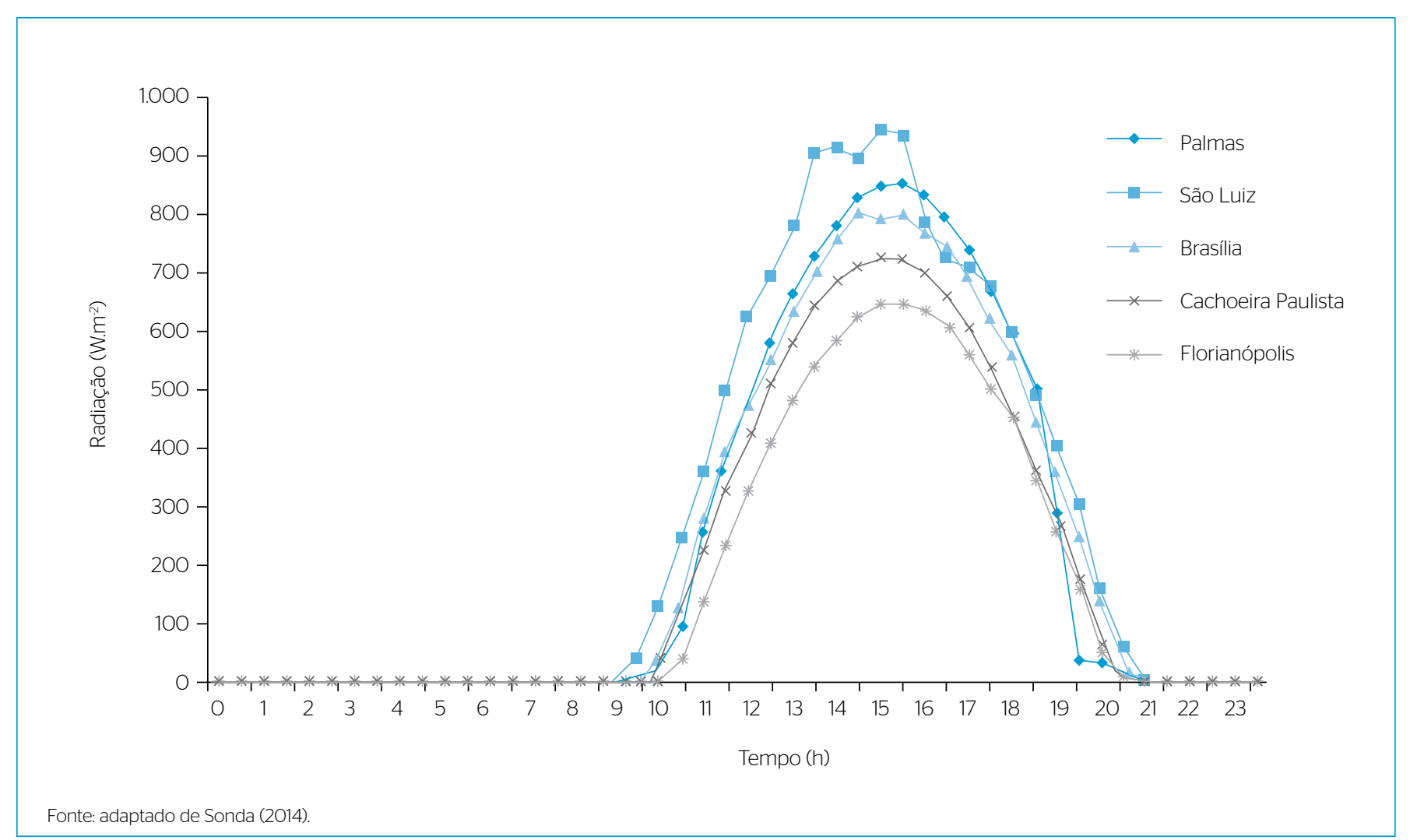

Figura 3 - Curvas de radiação de municípios por região. 
realizada uma simulação por um período de 6 dias com intervalos de 30 minutos. O fluxograma representado na Figura 4 demonstra a metodologia utilizada. Posteriormente, são descritas suas abordagens e seus parâmetros.

A Tabela 1 apresenta uma síntese das variáveis consideradas no modelo, respectivas unidades e fontes utilizadas. Inicialmente, devem ser informados dados relativos a localização geográfica e padrão de radiação, número de consumidores e padrão de consumo de água, características de sistemas de bombeamento, entre outros, conforme indicado na Figura 4.

De acordo com a localização geográfica, torna-se possível o conhecimento do perfil de radiação solar da região citada. Todavia, existe a opção de incluir curvas preexistentes de radiação solar e consumo de água, além de utilizar um sistema de bombeamento pré-definido.

Adiante, devem ser definidos o tipo de poço pretendido e os cenários diários quanto aos seguintes perfis de irradiância: ensolarado, parcialmente nublado, nublado ou chuvoso. Cada perfil possui um fator solar associado a horas de sol pleno (HSP): 1 para dia ensolarado, com 6 HSP; 0,71 para dia parcialmente nublado, com 4 HSP; 0,42 para dia nublado, com 2,5 HSP; e 0,17 para dia chuvoso, com 1 HSP (PINHO \& GALDINO, 2014). Ainda segundo o autor citado, o conceito de HSP é dado pelo número de horas de sol, em média diária, com uma intensidade de $1.000 \mathrm{~W} . \mathrm{m}^{-2}$. É equivalente à energia total diária incidente sobre a superfície em kWh.m ${ }^{-2}$.

Em função dos parâmetros mencionados acima, o modelo fornece o dimensionamento otimizado dos subsistemas (poço - quantidade e tipo; conjunto hidroenergético - painéis fotovoltaicos e bombas; reservatório - capacidade), a fim de garantir a melhor economia e assegurar o fornecimento de água almejado. Como resultados gráficos, são mostrados variação diária, em intervalos de meia hora, dos volumes remanescente no reservatório, volume excedente ou volume de falta, conforme ocorram.

\section{RESULTADOS E DISCUSSÃO}

Um aspecto de grande importância a ser considerado no dimensionamento de sistemas de bombeamento fotovoltaico é a variação do volume bombeado em função das variações diárias e horárias da radiação solar, sobretudo quando se projeta um sistema sem banco de baterias, porque o consumo de água também é variável ao longo do dia, afetando diretamente a capacidade do reservatório.

A Figura 5 ilustra o comportamento da vazão bombeada, no sistema piloto, em função da radiação solar, coletada por meio de um piranômetro, instalado próximo dos painéis fotovoltaicos, no mesmo plano de inclinação. Considerou-se um período de 6 dias, igualmente aplicado por Djurin, Margeta \& Glasnovic (2011),
Tabela 1 - Variáveis empregadas no cálculo do modelo.

\begin{tabular}{|c|c|c|}
\hline Variável & Unidade & Fonte \\
\hline Região, latitude e longitude & --- & Inf. \\
\hline Número de pessoas $(\mathrm{N})$ & hab. & Inf. \\
\hline Dias de autonomia (Da) & dias & Inf. \\
\hline $\begin{array}{l}\text { Fator de segurança falta de sol (Ffs); fator } \\
\text { sol (FS) }\end{array}$ & adim. & Inf. \\
\hline Altura geométrica total $(\mathrm{Hg})$ & metros $(\mathrm{m})$ & Inf. \\
\hline Comprimento de recalque $(\mathrm{L})$ & (m) & Inf. \\
\hline $\begin{array}{l}\text { Consumo médio per capita (q); padrão de } \\
\text { consumo (PC) }\end{array}$ & L.hab¹/dia & Inf. \\
\hline Altura do volume morto (Ym) & $(\mathrm{m})$ & Inf. \\
\hline $\begin{array}{l}\text { Porcentagem de enchimento do } \\
\text { reservatório (\%e) }\end{array}$ & $\%$ & Inf. \\
\hline Horas de sol pleno padrão (HSP padrão) & horas (h) & Inf. \\
\hline Vazão máxima do poço (Qpoço) & $m^{3} h^{-1}$ & Inf. \\
\hline Sistema painel + bomba (sistema P+B) & -- & Inf. ou Calc. \\
\hline Padrão de radiação (PR) & Wh.m ${ }^{-2}$ & Inf. ou Sel. \\
\hline Volume inicial do reservatório (Vo) & Litros (L) & Inf. ou Calc. \\
\hline Radiação total local (Rtl) & Wh.m ${ }^{2}$ & Inf. ou Sel. \\
\hline Horas de sol pleno local (HSP local)/zonas & horas & Sel. INPE \\
\hline Número de horas de sol (NS) & horas & Sel. \\
\hline Fator multiplicativo horário (Fmh) & adim. & Sel. \\
\hline $\begin{array}{l}\text { Volume morto }(\mathrm{Vm}) \text {; volume de } \\
\text { enchimento }(\mathrm{Ve})\end{array}$ & Litros (L) & Calc. \\
\hline $\begin{array}{l}\text { Volume útil do reservatório }(\mathrm{Vu}) \text {; volume } \\
\text { nominal }(\mathrm{Vn})\end{array}$ & Litros (L) & Calc. \\
\hline Radiação média local (Rml) & W. $m^{2}$ & Calc. \\
\hline Fator multiplicativo radiação solar (R) & adim. & Calc. \\
\hline Fator de multiplicação horário (Fm) & adim. & Calc. \\
\hline Diâmetro do reservatório (D) & metros $(\mathrm{m})$ & Calc. \\
\hline Maior índice de radiação (IRmáx) & Wh.m ${ }^{2}$ & Calc. \\
\hline Vazão de bombeamento média (Qbm) & $\left(L . h^{-1}\right)$ & Calc. \\
\hline $\begin{array}{l}\text { Vazão de bombeamento média local } \\
\text { (Qmed local) }\end{array}$ & $\left(L . h^{-1}\right)$ & Calc. \\
\hline Vazão de bombeamento máxima (Qmax) & $\left(L . h^{-1}\right)$ & Calc. \\
\hline Vazão para cálculo do diâmetro (Qd) & $\left(L . h^{-1}\right)$ & Calc. \\
\hline $\begin{array}{l}\text { Vazão de bombeamento média com } \\
\text { segurança (Qsmed) }\end{array}$ & $(L / h)$ & Calc. \\
\hline Vazão necessária para sistema (Qn) & $\left(L . h^{-1}\right)$ & Calc. \\
\hline Vazão nominal da bomba (Qb) & $\left(L . h^{-1}\right)$ & Calc. \\
\hline Diâmetro de recalque (Dr) & $(\mathrm{mm})$ & Calc. \\
\hline $\begin{array}{l}\text { Altura manométrica para a máxima altura } \\
\text { geométrica }(\mathrm{Hm})\end{array}$ & (m) & Calc. \\
\hline Vazão média (Qmed) & $\left(L . h^{-1}\right)$ & Calc. \\
\hline Volume bombeado (Vb) & $(\mathrm{L})$ & Calc. \\
\hline Altura de água no reservatório $(\mathrm{H})$ & (m) & Calc. \\
\hline Volume teórico do reservatório (Vt) & $(\mathrm{L})$ & Calc. \\
\hline Volume remanescente no reservatório (V) & $(\mathrm{L})$ & Calc. \\
\hline Volume excedente do reservatório (Vex) & $(\mathrm{L})$ & Calc. \\
\hline Volume de falta do reservatório (Vf) & $(\mathrm{L})$ & Calc. \\
\hline
\end{tabular}

Adim: adimensional; inf: informado; sel.: selecionado; calc: calculado; hab: habitantes. 
com padrões distintos de radiação solar diária, de aproximadamente 1,0 a 6,6 kWh.m ${ }^{-2}$. O período indicado compreende de 29 de outubro até 3 de novembro de 2015, sendo esses dados utilizados para uma simulação aplicando o modelo de dimensionamento proposto. Conforme observado na Figura 5, a vazão bombeada para o reservatório tem variação diretamente proporcional à radiação incidente no plano do painel fotovoltaico. Essa relação se torna mais clara na Tabela 2.

A relação da vazão diária bombeada $(\mathrm{Qb})$, medida (com $\mathrm{Hm}=10 \mathrm{~m}$, duas bombas operando em paralelo, sob as condições locais de radiação, está também medida), em função da vazão máxima dada pelo fabricante (Qmáx=11.200 litros/dia, mesmo tipo de associação de bombas, $\mathrm{Hm}=10 \mathrm{~m}$, radiação de 6.000 Wh. $\mathrm{m}^{-2}$ ), aumenta conforme a radiação diária, aspecto ilustrado pela Figura 6, também evidenciado por Hadj Arab et al. (1991). Essa figura exibe o gráfico de dispersão e a linha de tendência no período estudado, cujo valor de $\mathrm{R}^{2}$ encontrado foi de 0,9348 .
Com o intuito de averiguar a correlação dos dados anteriormente citados, utilizou-se o coeficiente de correlação por postos de Spearman, aplicando-se a Equação 1:

$\rho=1-\frac{6 \sum d^{2}}{n\left(n^{2}-1\right)}$

Em que:

$\mathrm{n}=$ número de amostras;

$\mathrm{d}=$ desvio padrão.

Após proceder à análise estatística, foi obtido $\rho=0,959$. O valor encontrado expressa uma boa correlação positiva, com os postos das duas variáveis, radiação total diária (Wh. $\mathrm{m}^{-2} / \mathrm{dia}$ ) e rendimento da bomba (Qb/Qmáx). É possível observar, na Figura 6, que, mesmo para valores de radiação total diária de $6.000 \mathrm{Wh} . \mathrm{m}^{-2}$, a relação entre a vazão diária bombeada (medida) e a indicada pelo fabricante (de catálogo) é inferior à unidade. Isso se deve ao fato de que os dados de catálogo, nesse caso, se referem a um período de radiação diária

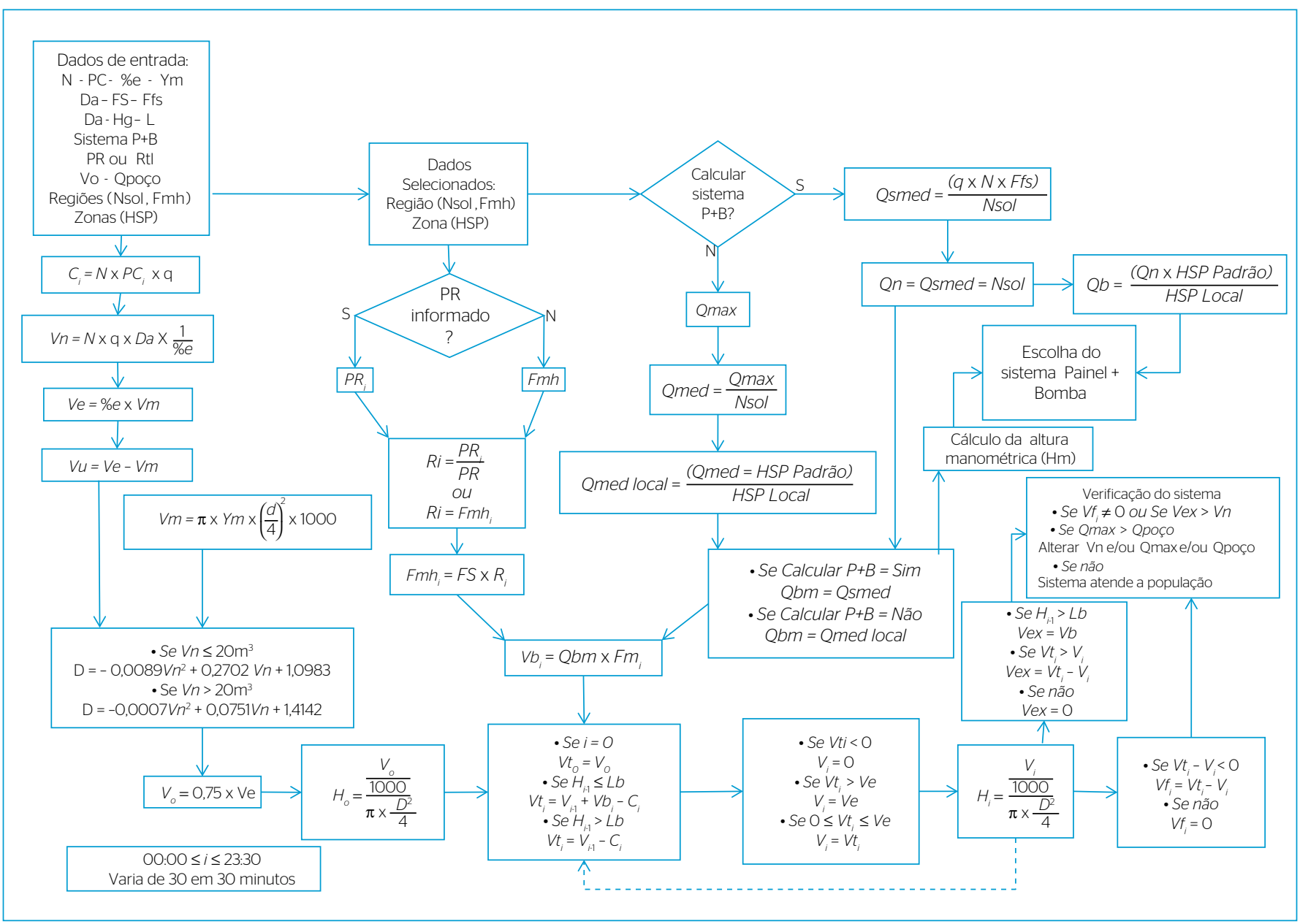

Figura 4 - Fluxograma de cálculo da capacidade de água do reservatório. 
de 6 horas com radiação de 1.000 Wh.m ${ }^{-2}$. Na prática, essa situação não ocorreu, conforme ilustra a Figura 5, mostrando a influência do padrão de radiação sobre o desempenho do sistema de bombeamento.

Para exemplificar a aplicabilidade do modelo, foi realizada uma simulação sob as seguintes condições: atendimento a 10 pessoas; consumo médio per capita q=240,0 $\mathrm{l}_{\mathrm{hab}}{ }^{-1}$ /dia; padrão de consumo conforme Figura 7 (baseado em levantamento feito em uma propriedade rural); padrão de radiação solar observado nos meses de outubro e novembro.

O período avaliado, ao invés de em função dos meses de inverno, conforme descrito em Pinho \& Galdino (2014), foi em virtude de terem sido observadas as menores médias registradas de radiação solar diária na região. $\mathrm{O}$ mês de outubro apresentou a maior precipitação mensal (313 mm) e a menor radiação anual (2.946 Wh.m $\left.{ }^{-2}\right)$. A forte influência do El niño no Hemisfério Sul pode ser uma das causas da anomalia observada nos padrões de radiação.

Foi testado o reservatório do sistema piloto, com capacidade nominal de $5.000 \mathrm{~L}$, e altura do volume morto de $0,12 \mathrm{~m}$, valor estabelecido em função da posição da tubulação de saída de água para consumo, e seu diâmetro, $32 \mathrm{~mm}$. O resultado dessa simulação está ilustrado na Figura 8.

É possível observar na Figura 8 que, em alguns momentos, existe maior aderência entre o nível previsto pelo modelo e o medido. Nos períodos com bombeamento, a diferença pode ser explicada pelo fato de, no modelo, em cada intervalo de meia hora, a radiação ser considerada constante, o que dificilmente acontece na natureza. Já nos períodos em que não há bombeamento, a diferença pode ser associada ao consumo, medido (variável) e registrado pelo modelo (fixo/constante). No período entre $23 \mathrm{~h}$ do dia 2 de novembro e $10 \mathrm{~h}$ do dia 3 de novembro, o nível de água no reservatório igualou-se ao do volume morto. Observando o padrão de consumo (Figura 7), é possível deduzir que houve uma falta da ordem de $450 \mathrm{~L}$ de água, relativa ao consumo no intervalo considerado, decorrente da ausência ou insuficiência de sol para acionar o sistema de bombeamento.

Após o processo de otimização, o modelo indica o volume mínimo do reservatório necessário, considerando, entre outros aspectos, o número de dias de autonomia e a tolerância a faltas. Djurin, Margeta \& Glasnovic (2011) consideram dois dias de autonomia como um valor muito aceitável.

A escolha final do tipo e da capacidade do reservatório deve ser feita pelo usuário, em função das opções disponíveis tanto em termos construtivos como de custos. O modelo não incorpora a informação da existência ou não de reservatório nas residências, contudo essa prática é bastante comum no Brasil. Isso pode significar um fator de segurança sobre o sistema dimensionado - na prática, pelo menos um dia a mais de autonomia se todos os reservatórios estiverem cheios no início de

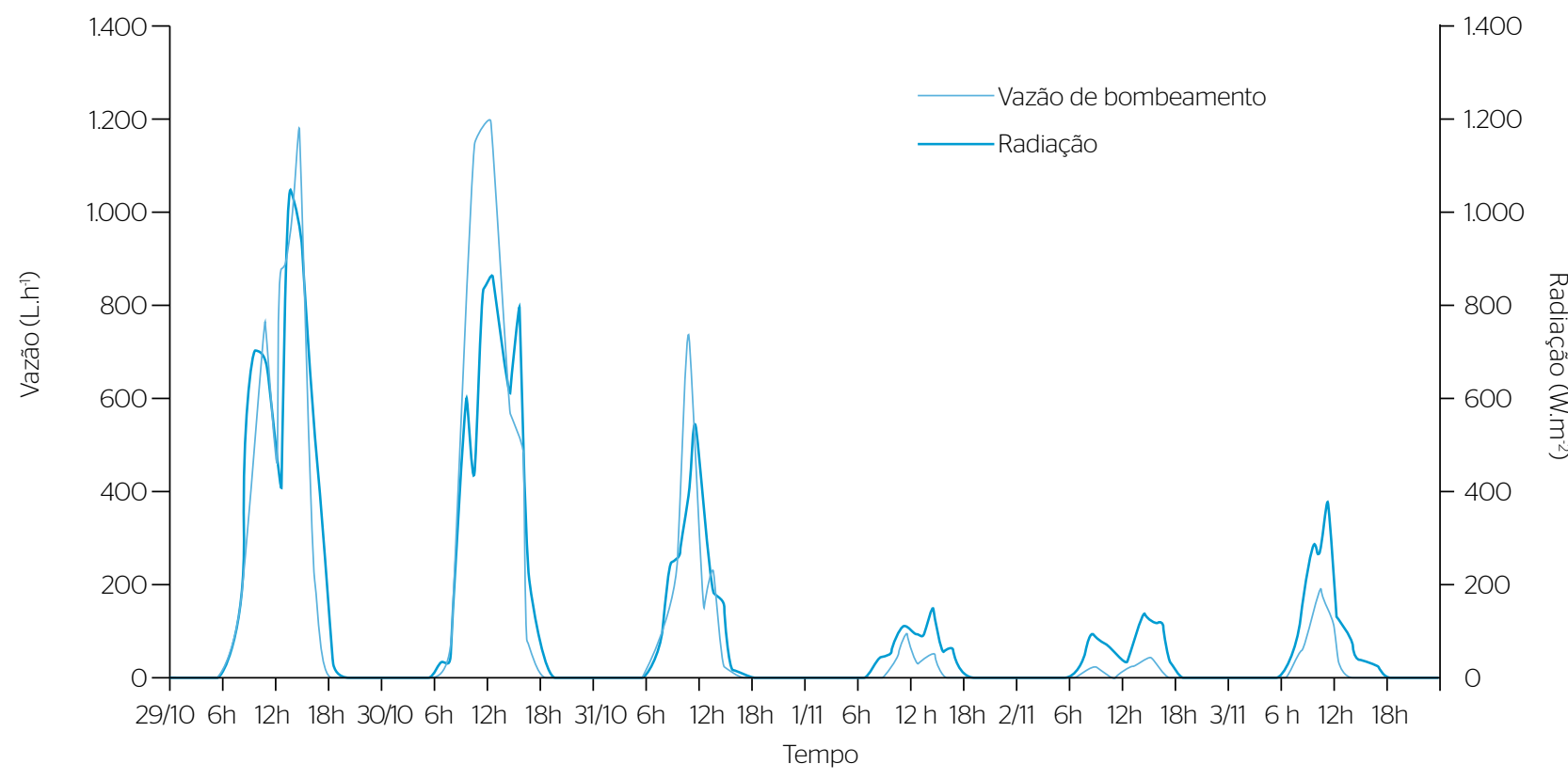

Figura 5 - Gráfico de vazão e radiação em função do tempo. 
um período de baixa radiação. Nessa perspectiva, o resultado da simulação ilustrado pela Figura 8 poderia não significar, necessariamente, falta de água nas residências atendidas pelo sistema.

\section{CONCLUSÕES}

Considerando o arranjo experimental utilizado e demais características do local avaliado, é possível estabelecer as seguintes conclusões:

- o padrão diário de radiação influencia de forma determinante no desempenho do sistema de bombeamento fotovoltaico, sendo esse um fator a se considerar na estimativa dos volumes diários bombeados;

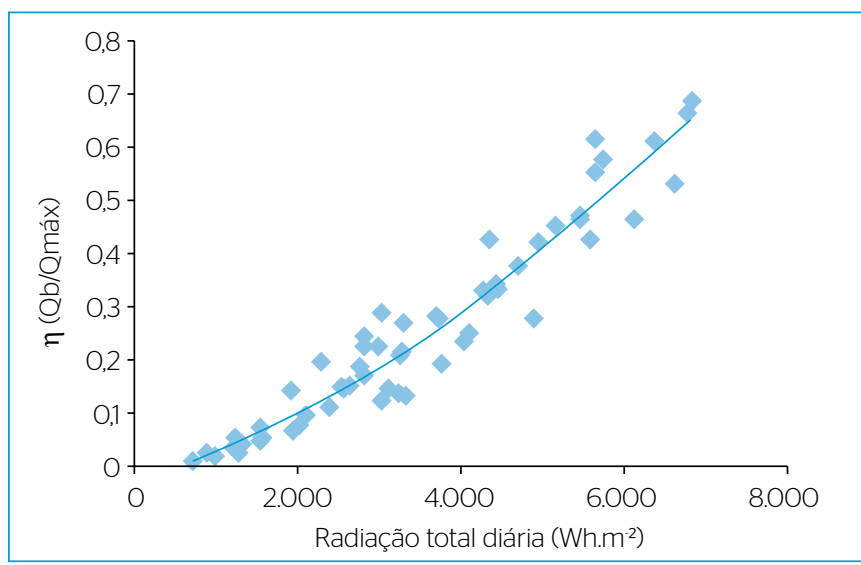

Figura 6 - Rendimento do bombeamento em função da radiação diária.

Tabela 2 - Relação da vazão de bombeamento pela vazão dada pelo fabricante.

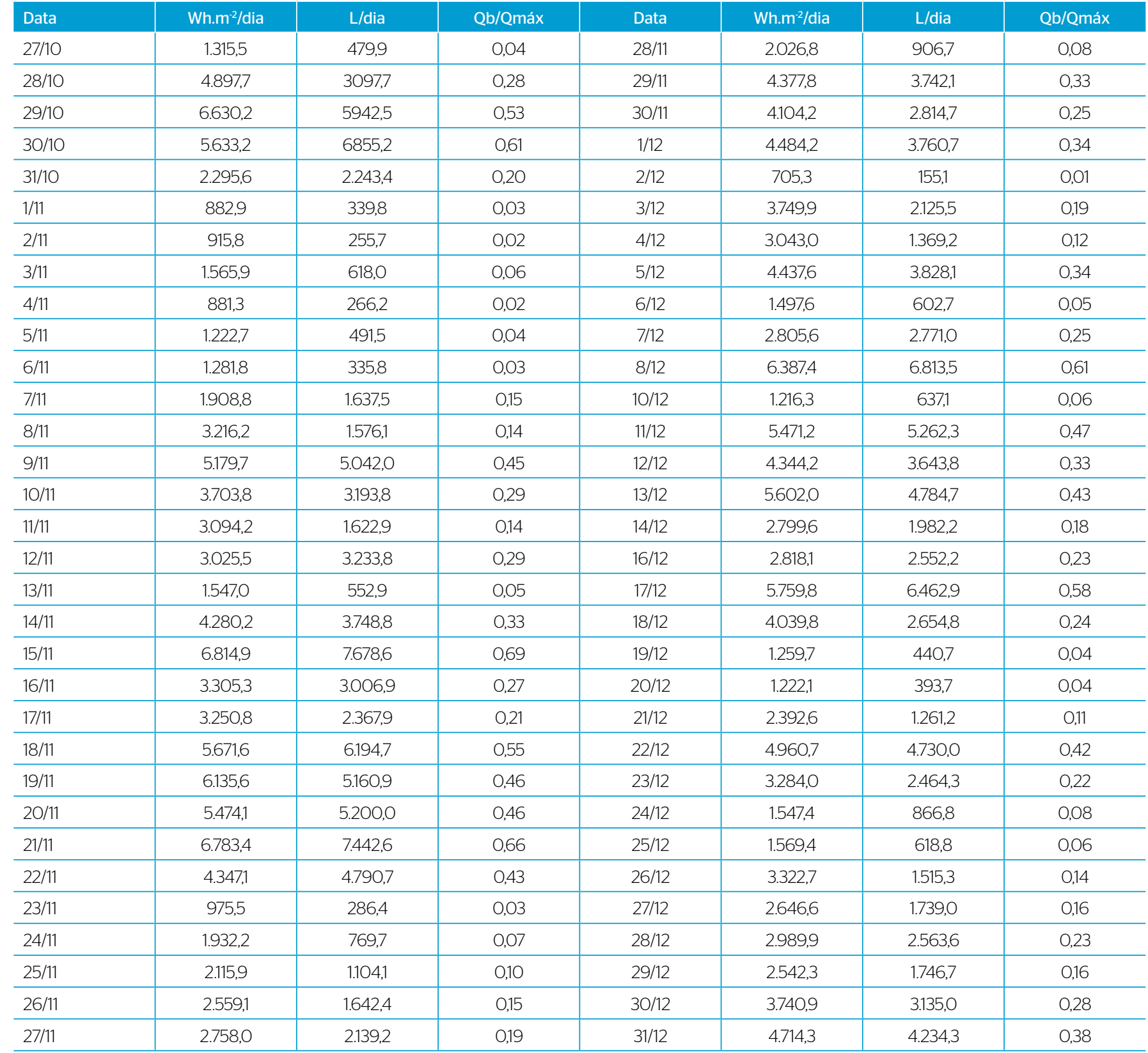




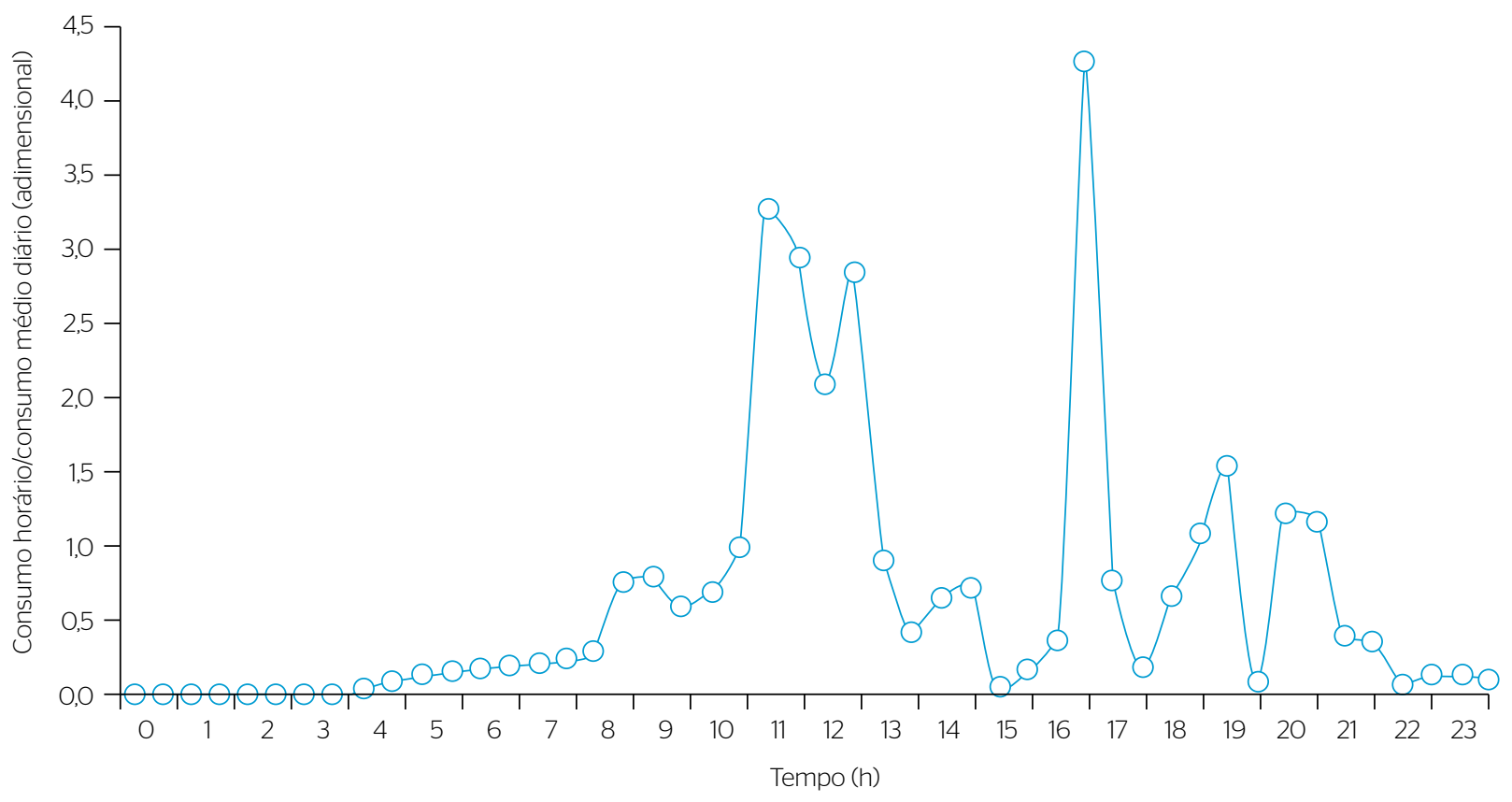

Figura 7 - Padrão de consumo de água diário.

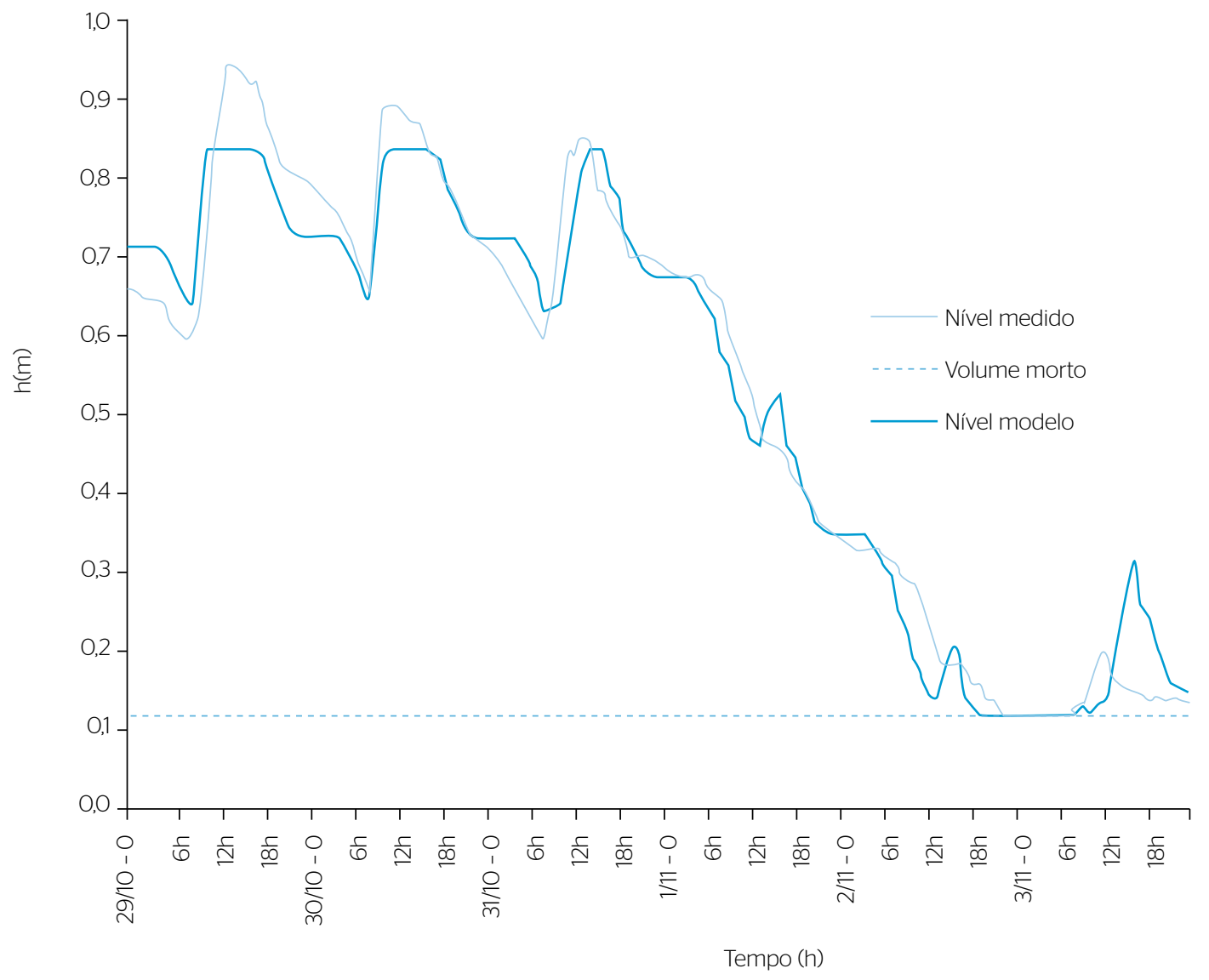

Figura 8 - Nível de água no reservatório previsto pelo modelo e medido. 
- o padrão de consumo não pode ser excluído da análise de capacidade do reservatório, notadamente em sistemas como o experimentado, sem banco de baterias, pois os consumos fora de horários de bombeamento podem ser significativos em termos de volume;

- o modelo proposto se mostrou apropriado para a simulação de desempenho do sistema de bombeamento fotovoltaico estudado. Adicionalmente, com o fornecimento dos dados de radiação solar e padrão de consumo de água, torna-se possível estabelecer uma solução otimizada para um determinado sistema, considerando a capacidade do reservatório, do poço de produção e do sistema de bombeamento utilizado.

Cabe ressaltar que o modelo possui algumas limitações, como a aleatoriedade dos dados de radiação solar, considerados constantes no modelo e variáveis na realidade. Tal diferença influencia no volume de bombeamento registrado pelo modelo e medido.

Os resultados das experimentações, após análises, apresentam uma visão futura do sistema. As informações geradas auxiliam nas tomadas de decisão, necessárias no momento presente, e contribuem para uma melhor compreensão do sistema a ser implantado.

\section{REFERÊNCIAS}

ANIS, W.R.; NORB, M.A. (1994) Optimum design of a photovoltaic powered pumping system. Journal of Power Sources, v. 50, p. 1-9. https://doi.org/10.1016/0378-7753(93)01882-I

BOITRAGO, S.A.; SANTOS, S.S.; GONÇALVES, A.H.R.; CANELA, M.A.R. (2016) Captação de água por sistema fotovoltaico para consumo humano na localidade rural ilha Caiapós, São Romão - Minas Gerais. Reec - Revista Eletrônica de Engenharia Civil, Goiânia, v. 12, n. 2, p. 43-52. http://dx.doi.org/10.5216/reec.v12i2.37490

DJURIN, B.; MARGETA J.; GLASNOVIC, Z. (2011) PV Power for Water Urban Supply. International Journal of Energy Science, v. 1, n. 3, p. 131-139.

GAD, H.E. (2009) Performance prediction of a proposed water pumping system at South Sinai, Egypt climate conditions. In: INTERNATIONAL WATER TECHNOLOGY CONFERENCE, 13., Hurghada, Egito. Proceedings... p. 739-752.

HADJ ARAB, A.; CHENLO, F.; MUKADAM, K.; BALENZATEGUI, J.L. (1991) Performance of PV water pumping systems. Renew Energy, v. 18, p. 191-204. http://dx.doi.org/10.1016/SO960-1481(98)00780-0

KOLHE, M.; JOSHI, J.C.; KOTHARI, D.P. (2004) Performance analysis of a direct coupled photovoltaic water pumping system. IEEE Transactions on Energy Conversion, v. 19, n. 3. https://doi.org/10.1109/TEC.2004.827032

LOXSOM, F.; DURONGKAVEROJ, P.D. (1994) Estimating the performance of a photovoltaic water pumping system. Sol Energy, v. 52, p. 215-219. https://doi.org/10.1016/0038-092X(94)90071-X
PEREIRA, E.B.; MARTINS, F.R.; ABREU, S.L.; RÜTHER, R. (2006) Atlas brasileiro de energia solar. São José dos Campos: Instituto Nacional de Pesquisas Espaciais (INPE). 60 p. Disponível em: <http://ftp.cptec.inpe.br/labren/publ//ivros/brazil_solar_atlas_R1.pdf>. Acesso em: 04 nov. 2015.

PINHO, J.T.; GALDINO, M.A. (2014) Manual de Engenharia para Sistemas Fotovoltaicos. Rio de Janeiro: Grupo de Trabalho de Energia Solar (GTES), Centro de Pesquisas de Energia Elétrica (CEPEL), Centro de Referência para Energia Solar e Eólica Sérgio de Salvo Brito (CRESESB). 530p.

SERVIÇO DE ORGANIZAÇÃO NACIONAL DE DADOS AMBIENTAIS (SONDA). (2014) Estações Sonda. Disponível em: <http://sonda.ccst. inpe.br/infos/index.html>. Acesso em: 25 ago. 2015.

SETIAWAN, A.A.; PURWANTO, D.H.; PAMUJI, D.S.; HUDA, P.N. (2014) Development of a Solar Water Pumping System in Karsts Rural Area Tepus, Gunungkidul through Student Community Services. Energy Procedia, Yogyakarta, v. 47, p. 7-14. http://dx.doi. org/10.1016/j.egypro.2014.01.190

TIBA, C. (Org.). (2000) Atlas Solarimétrico do Brasil: banco de dados solarimétricos. Recife: Editora Universitária da UFPE. 111 p.

UNITED NATIONS. (2015) Transforming our world: The 2030 Agenda for Sustainable Development. United Nations. Disponível em: <https://sustainabledevelopment.un.org/post2015/ transformingourworld/publication>. Acesso em: 11 jan. 2016. 